May 1998

IFP-761-UNC

\title{
Cosmic Background Radiation Temperature Anisotropy: Position of First Doppler Peak
}

\author{
Paul H. Frampton, Y.Jack Ng and Ryan Rohm \\ Department of Physics and Astronomy, \\ University of North Carolina, Chapel Hill, NC 27599-3255
}

\begin{abstract}
The purpose of the Cosmic Background Radiation (CBR) experiments is to measure the temperature anisotropy via the autocorrelation function. The partial wave $l_{1}$ corresponding to the first Doppler peak caused by baryonphoton oscillations at the surface of last scattering depends on the present density $\Omega_{0}$ and the cosmological constant contribution $\Omega_{\Lambda}$. We discuss this dependence on the basis of perspicuous figures.
\end{abstract}

Typeset using REVTEX 


\section{CBR TEMPERATURE ANISOTROPY.}

Although the Cosmic Background Radiation (CBR) was first discovered over thirty years ago [1], the detection of its temperature anisotropy waited until 1992 when the Cosmic Background Explorer (COBE) satellite provided its impressive experimental support [2, 3] for the Big Bang model. In particular, the COBE results were consistent with a scale-invariant

spectrum of primordial scalar density perturbations [4] such as might be generated by quantum fluctuations during an inflationary period. [8 10

This discovery of temperature anisotropy in the CBR has inspired many further experiments which will be sensitive to smaller angle anisotropies than the COBE satellite was (about $1^{\circ}$ ). NASA has approved the flight of a satellite mission, the Microwave Anisotropy Probe (MAP) in the year 2000 and ESA has agreed to a more accurate later experiment called the Planck Surveyor. The expected precision of these measurements implies that the angular dependence of the temperature anisotropy will be known sufficiently well that the location of the first accoustic (Doppler) peak, and possibly subsequent ones, will be resolved.

Although the hot big bang theory is supported by at least three major triumphs: the expansion of the universe, the cosmic background radiation and the nucleosynthesis calculations, it leaves unanswered several questions. The most important unanswered questions are the horizon and flatness issues.

When the CBR last scattered, the age of the universe was about 100,000 years compared to its present age of some 10 billion years. As we shall see, the horizon size at the recombination time subtends now an angle of about $(1 / 208)$ of $\pi$ radians. On the celestial sphere there are therefore approximately 40,000 causally disconnected regions. Nevertheless, these different regions have a uniform CBR temperature to an accuracy of better than one part in $10^{5}$. This is the horizon problem.

The flatness problem may be understood from the cosmological equation

$$
\frac{k}{R^{2}}=(\Omega-1) \frac{\dot{R}^{2}}{R^{2}}
$$


Evaluating Eq.(1) at an arbitrary time $t$ and dividing by the same relation at the present time $t=t_{0}$ and using $R \sim \sqrt{t} \sim T^{-1}$ gives

$$
(\Omega-1)=4 H_{0}^{2} t^{2} \frac{T^{2}}{T_{0}^{2}}\left(\Omega_{0}-1\right)
$$

For high densities we write

$$
\frac{\dot{R}^{2}}{R^{2}}=\frac{8 \pi G \rho}{3}=\frac{8 \pi G g a T^{4}}{6}
$$

where $a$ is the radiation constant and $\mathrm{g}$ is the effective number of degrees of freedom. This leads to the relation between time and temperature, after substituting the numerical values $\left[a=7.56 \times 10^{-9} \mathrm{ergm}^{-3} \mathrm{~K}^{-4} ; G / \mathrm{c}^{2}=0.742 \times 10^{-30} \mathrm{~m} / \mathrm{g} ; H_{0}=100 h_{0} \mathrm{~km} / \mathrm{s} / \mathrm{Mpc}=\right.$ $\left.3.25 \times 10^{-18} h_{0} s^{-1}\right]$

$$
t(\text { seconds })=\left(2.42 \times 10^{-6}\right) g^{-1 / 2} T_{G e V}^{-2}
$$

Combining Eq.(2) with Eq.(4) leads to

$$
(\Omega-1)=3.64 \times 10^{-21} h_{0}^{2} g^{-1} T_{G e V}^{-2}\left(\Omega_{0}-1\right)
$$

Given the proximity of $\Omega_{0}$ to unity, we then deduce that $\Omega$ at, for example, $T=1 \mathrm{MeV}(t \sim$ 1second) must be equal to one within one part in $10^{14}$ ! Otherwise the resultant cosmology will be incompatible with the present situation of our universe. This extraordinary finetuning is the flatness problem.

The goal [11]17 of the CBR experiments is to measure the temperature autocorrelation function. The fractional temperature perturbation as a function of the direction $\hat{\mathbf{n}}$ is expanded in spherical harmonics

$$
\frac{\Delta T(\hat{\mathbf{n}})}{T}=\sum_{l m} a_{(l m)} Y_{l m}(\hat{\mathbf{n}})
$$

and the statistical isotropy and homogeneity of the universe imply that the coefficients have expectation values

$$
<\left(a_{(l m)}\right)^{*} a_{\left(l^{\prime} m^{\prime}\right)}>=C_{l} \delta_{l l^{\prime}} \delta_{m m^{\prime}}
$$


The plot of $C_{l}$ versus $l$ is expected to reflect oscillations in the baryon-photon fluid at the surface of last scatter. In particular, the first Doppler peak should be at the postion $l_{1}=\pi / \Delta \theta$ where $\Delta \theta$ is the angle now subtended by the horizon at the time of the last scattering, namely the recombination time corresponding to a red shift $z_{t} \sim 1,100$.

The horizon and flatness problems described above can both be solved by the inflation scenario which has the further prediction that $\Omega_{0}=1$ if the cosmological constant vanishes or more generally that $\Omega_{0}+\Omega_{\Lambda}=1$ if the cosmological constant does not vanish.

The question we address here is restricted to the question of how much the value of $l_{1}$ alone - likely to be accurately determined in the next few years - will tell us about the values of the cosmic parameters $\Omega_{0}$ and $\Omega_{\Lambda}$ ?

In Section 2, the case $\Lambda=0$ is discussed. In Section 3, there is the more general case; and finally in Section 4 there is discussion of the Figures derived. 


\section{THE SPECIAL CASE $\Lambda=0,0<\Omega_{0}<1$}

When the cosmological constant vanishes, the Einstein-Friedmann cosmological equations can be solved analytically (not the case, in general, when $\Lambda \neq 0$ ). So we shall begin by doing this special case explicitly. It gives rise to the well-known result that the position of the first Doppler peak (partial wave $l_{1}$ ) expected in the partial-wave analysis depending on the present matter-energy density $\Omega_{0}$ (for $\Lambda=0$ ) according to $l_{1} \sim 1 / \sqrt{\Omega_{0}}$ [13, [17]. We shall show in the next section how in the general case with $\Lambda \neq 0$ there is a rather serious "comic confusion" in disentangling the value of $\Omega_{0}$ from the position $l_{1}$ of the first Doppler peak.

Let us use the metric:

$$
d s^{2}=d t^{2}-R^{2}\left[d \Psi^{2}+\sinh ^{2} \Psi d \theta^{2}+\sinh ^{2} \Psi \sin ^{2} \theta d \phi^{2}\right]
$$

For a geodesic $d s^{2}=0$ and, in particular,

$$
\frac{d \Psi}{d t}=\frac{1}{R}
$$

Einstein's equation reads

$$
\left(\frac{\dot{R}}{R}\right)^{2}=\frac{8 \pi}{3} G \rho+\frac{1}{R^{2}}
$$

where we take curvature $k=-1$. Let us define:

$$
\Omega_{0}=\frac{8 \pi G \rho_{0}}{3 H_{0}^{2}} ; \rho=\rho_{0}\left(\frac{R_{0}}{R}\right)^{3} ; a=\Omega_{0} H_{0}^{2} R_{0}^{3}
$$

Then from Eq.(10) we find that

$$
\dot{R}^{2} R^{2}=R^{2}+a R
$$

and so it follows that

$$
\frac{d \Psi}{d R}=\frac{d \Psi}{d t}\left(\frac{d R}{d t}\right)^{-1}=\frac{1}{\dot{R} R}=\frac{1}{\sqrt{R^{2}+a R}}
$$

Since $\Psi_{0}=0$, the value at time $t$ can be computed from the integral 


$$
\Psi_{t}=\int_{R_{t}}^{R_{0}} \frac{d R}{\sqrt{(R+a / 2)^{2}-(a / 2)^{2}}}
$$

This can be performed easily with the substitution $R=\frac{1}{2} a(\cosh V-1)$ to give the result:

$$
\Psi_{t}=\cosh ^{-1}\left(\frac{2 R_{0}}{a}+1\right)-\cosh ^{-1}\left(\frac{2 R_{t}}{a}+1\right)
$$

¿From Eq.(10) evaluated at $t=t_{0}$ we see that

$$
\frac{1}{a}=\frac{1-\Omega_{0}}{R_{0} \Omega_{0}}
$$

and so, using $\sinh \left(\cosh ^{-1} x\right)=\sqrt{x^{2}-1}$ in Eq.(15) gives now

$$
\sinh \Psi_{t}=\left(\sqrt{\left(\frac{2\left(1-\Omega_{0}\right)}{\Omega_{0}}+1\right)^{2}-1}\right)\left(\frac{2 R_{t}}{a}+1\right)-\left(\sqrt{\left(\frac{2\left(1-\Omega_{0}\right) R_{t}}{\Omega_{0} R_{0}}+1\right)^{2}-1}\right)\left(\frac{2 R_{0}}{a}+1\right)
$$

The position of the first Doppler peak depends on the angle subtended by the horizon size at the time $t$ equal to the recombination time. This corresponds to the distance $\left(H_{t}\right)^{-1}$. According to the metric of Eq.(8) the angle subtended is

$$
\Delta \theta=\frac{1}{H_{t} R_{t} \sinh \Psi_{t}}
$$

and the position of the first Doppler peak corresponds to the partial wave $l_{1}$ given by

$$
l_{1}=\frac{\pi}{\Delta \theta}=\pi H_{t} R_{t} \sinh \Psi_{t}
$$

Now the red-shift at recombination is about $z_{t}=1100 \simeq\left(R_{0} / R_{t}\right) \gg 1$ so we may approximate in Eq.(17) to find

$$
\sinh \Psi_{t} \simeq \frac{2 \sqrt{1-\Omega_{0}}}{\Omega_{0}}
$$

Using $H_{t}^{2}=8 \pi G \rho / 3+1 / R^{2} \simeq \Omega_{0} H_{0}^{2}\left(R_{0} / R_{t}\right)^{3}$ gives

$$
l_{1}(\Lambda=0)=\frac{2 \pi}{\sqrt{\Omega_{0}}} z_{t}^{1 / 2}
$$

In particular, if $\Omega_{0}=1$ and $\Lambda=0$, one has $l_{1} \simeq 208.4$. If $l_{1}$ does have this value empirically it will favor this simplest choice, although as we shall see in the following subsection even here the conclusion has ambiguities.

In Fig. 1 we plot $l_{1}$ versus $\Omega_{0}$ for the particular case of $\Omega_{\Lambda}=0$. 


\section{THE GENERAL CASE: $0 \leq \Omega_{0}<2 ; 0 \leq \Omega_{\Lambda}<1$}

For the general case of $0 \leq \Omega_{\Lambda}<2 ; 0<\Omega_{0}<1$ we use the more general Einstein cosmological equation:

$$
\dot{R}^{2} R^{2}=-k R^{2}+a R+\Lambda R^{4} / 3
$$

where $a=\Omega_{0} H_{0}^{2} R_{0}^{3}$. We define

$$
\Omega_{0}=\frac{8 \pi G \rho_{0}}{3 H_{0}^{2}} ; \Omega_{\Lambda}=\frac{\Lambda}{3 H_{0}^{2}} ; \Omega_{C}=\frac{-k}{H_{0}^{2} R_{0}^{2}}
$$

Substituting $R=R_{0} r$ and $w=1 / r$ now gives rise to the integral [18] for $\Psi_{t}$

$$
\Psi_{t}=\sqrt{\Omega_{C}} \int_{1}^{\infty} \frac{d w}{\sqrt{\Omega_{\Lambda}+\Omega_{C} w^{2}+\Omega_{0} w^{3}}}
$$

in which $\Omega_{\Lambda}+\Omega_{C}+\Omega_{0}=1$.

Consider first the case of an open universe $\Omega_{C}>0$. Then

$$
l_{1}=\pi H_{t} R_{t} \sinh \Psi_{t}
$$

We know that

$$
\begin{aligned}
H_{t}^{2} & =\left(\frac{\dot{R}_{t}}{R_{t}}\right)^{2}=\frac{8 \pi G \rho}{3}+\frac{\Lambda}{3}+\frac{1}{R_{t}^{2}} \\
& =H_{0}^{2}\left[\Omega_{0}\left(\frac{R_{0}}{R_{t}}\right)^{3}+\Omega_{\Lambda}+\left(\frac{R_{0}}{R_{t}}\right)^{2} \Omega_{C}\right]
\end{aligned}
$$

Since $R_{0} \gg R_{t}$ we may approximate:

$$
H_{t} \simeq\left(\frac{R_{0}}{R_{t}}\right)^{3 / 2} H_{0} \sqrt{\Omega_{0}}
$$

and hence

$$
H_{t} R_{t}=\left(\frac{R_{0}}{R_{t}}\right)^{1 / 2} \sqrt{\frac{\Omega_{0}}{\Omega_{C}}}
$$

It follows that for this case $\Omega_{C}>0$ that

$$
l_{1}=\pi \sqrt{\frac{\Omega_{0}}{\Omega_{C}}}\left(\frac{R_{0}}{R_{t}}\right)^{1 / 2} \sinh \left(\sqrt{\Omega_{C}} \int_{1}^{\infty} \frac{d w}{\sqrt{\Omega_{\Lambda}+\Omega_{C} w^{2}+\Omega_{0} w^{3}}}\right)
$$


For the case $\Omega_{C}<0(k=+1)$ we simply replace $\sinh$ by $\sin$ in Eq. (29). Finally, for the special case $\Omega_{C}=0$, the generalized flat case favored by inflationary cosmologies, Eq.(29) simplifies to:

$$
l_{1}=\pi \sqrt{\Omega_{0}}\left(\frac{R_{0}}{R_{t}}\right)^{1 / 2} \int_{1}^{\infty} \frac{d w}{\sqrt{\Omega_{\Lambda}+\Omega_{0} w^{3}}}
$$

In Fig. 2 we plot the value of $l_{1}$ versus $\Omega_{0}$ for the case $\Omega_{C}=0$ (flat spacetime). The contrast with Fig 1 is clear: whereas $l_{1}$ increases with decreasing $\Omega_{0}$ when $\Omega_{\Lambda}=0$ (Fig. 1) the opposite behaviour occurs when we constrain $\Omega_{\Lambda}=1-\Omega_{0}$ (fig.2).

With $\Omega_{0}$ and $\Omega_{\Lambda}$ unrestricted there are more general results. In Fig. 3 , we display iso-l lines on a $\Omega_{0}-\Omega_{\Lambda}$ plot. The iso-l lines are (from right to left) for the values $l_{1}=150,160,170,180,190,200,210,220,230,240,250,260,270$ respectively. One can see that from the position $\left(l_{1}\right)$ only of the first Doppler peak there remains a serious ambiguity of interpretation without further information.

In Fig, 4 , there is a three dimensional rendition of the value of $l_{1}$ versus the two variables $\Omega_{0}$ and $\Omega_{\Lambda}$. 


\section{DISCUSSION OF COSMIC PARAMETER AMBIGUITIES.}

Let us now turn to an interpretation of our Figures, from the point of view of determining the cosmic parameters.

In the case where $\Lambda=\Omega_{\Lambda}=0$, Fig.1. is sufficient. In this case, there is the well-known dependence [13,17] $l_{1}=(208.4) / \sqrt{\Omega_{0}}$ illustrated in Fig.1. It would be straightforward to determine $\Omega_{0}$ with an accuracy of a few percent from the upcoming measurements.

Of course there is a strong theoretical prejudice towards $\Lambda=0$. But no underlying symmetry principle is yet known. If $\Omega_{\Lambda} \neq 0$, one knows that it is not bigger than order one; this is very many orders of magnitude smaller than expected [19 from the vacuum energy arising in spontaneous breaking of symmetries such as the electroweak group $S U(2) \times U(1)$.

Nevertheless, recent observations of high redshift Type 1a supernovae have led to the suggestion of an increasing Hubble parameter [20,21]. An interpretation of this is that the cosmological constant is non-zero, possibly $\Omega_{\Lambda} \simeq 0.7$ but is still consistent with $\Omega_{0}=$ $1-\Omega_{\Lambda}$. It should be added that these conclusions are quite controversial and await further verification. But these results are enough to motivate a full consideration of non-zero values of $\Omega_{\Lambda}$.

Thus we come to Fig. 2 which depicts the $\Omega_{0}$ dependence of $l_{1}$ when $\Omega_{0}+\Omega_{\Lambda}=1$ is held fixed as in a generalized flat cosmology that could arise from inflation. We notice that here $l_{1}$ decreases as $\Omega_{0}$ decreases from $\Omega_{0}=1$, the opposite behaviour to Fig. 1 . Thus even the sign of the shift of $l_{1}$ from $l_{1}=208.4$ depends on the size of $\Lambda$.

It is therefore of interest to find what are the contours of constant $l_{1}$ in the $\Omega_{0}-\Omega_{\Lambda}$ plane. These iso-l lines are shown in Fig. 3 for $l_{1}=150, \ldots, 270$ in increments $\Delta l_{1}=10$. If we focus on the $l_{1}=210$ contour (the seventh contour from the left in Fig. 3) as an example, we see that while this passes close to the $\Omega_{0}=1, \Lambda=0$ point it also tracks out a line naturally between those shown in Figs. 1 and 2 (actually somewhat closer to the latter than the former).

Finally, Fig. 4 gives a three-dimensional rendition which includes Figures 1 to 3 as special 
cases and provides a visualisation of the full functional dependence of $l_{1}\left(\Omega_{0}, \Omega_{\Lambda}\right)$.

Our main conclusion is that the position $l_{1}$ of the first Doppler peak will define the correct contour in our iso-l plot, Fig. 3. More information will be necessary to determine $\Omega_{0}$ and the validity of inflation.

We thank Eric Carlson of Wake Forest University for useful discussions, and Masayasu Harada for help. This work was supported in part by the US Department of energy under Grant No. DE-FG05-85ER-40219.

\section{Note Added.}

After completing this paper, three very recent papers having some overlap with our work were brought to our attention:

M. White. astro-ph/9802295; M. Tegmark, D.J. Eisenstein, W. Hu and R.G. Kron. astro-ph/9805117; C.H. Lineweaver. astro-ph/9805320. 


\section{REFERENCES}

[1] A.A. Penzias and R.W. Wilson, Ap. J. 142, 419 (1965).

[2] G.F. Smoot et al., Ap. J. Lett. 396, L1 (1992).

[3] K. Ganga et al., Ap.J. 410, L57 (1993).

[4] J.M. Bardeen, P.J. Steinhardt and M.S. Turner, Phys. Rev. D28, 679 (1983).

[5] A.A. Starobinsky, Phys. Lett. B117, 175 (1982).

[6] A.H. Guth and S.-Y. Pi, Phys. Rev. Let. 49, 1110 (1982).

[7] S.W. Hawking, Phys. Lett. B115, 295 (1982).

[8] A.H. Guth, Phys. Rev. D28, 347 (1981).

[9] A.D. Linde, Phys. Lett. B108, 389 (1982).

[10] A. Albrecht and P.J. Steinhardt, Phys. Rev. Lett. 48, 1220 (1982).

[11] R.L. Davis, H.M. Hodges, G.F. Smoot, P.J. Steinhardt and M.S. Turner, Phys. Rev. Lett. 69, 1856 (1992).

[12] J.R. Bond, R. Crittenden, R.L. Davis, G. Efstathiou and P.J. Steinhardt, Phys. Rev. Lett. 72, 13 (1994).

[13] P.J. Steinhardt, Int. J. Mod. Phys. A10, 1091 (1995).

[14] A. Kosowsky, M. Kamionkowski, G. Jungman and D.N. Spergel, Determining Cosmological Parameters from the Microwave Background. Talk at 2nd Symposium on Critique of the Sources of Dark Matter in the Universe, Santa Monica, CA, Feb 14-16, 1996; Nucl. Phys. Proc. Suppl. 51B, 49 (1996).

[15] M. Kamionkowski and A. Loeb, Phys. Rev. D56, 4511 (1997).

[16] M. Kamionkowski, Cosmic Microwave Background Tests of Inflation. 
To be published in Proceedings of the 5th International Workshop on Topics in Astroparticle and Underground Physics (TAUP97), Gran Sasso, Italy, September 7-11, 1997.

[17] M. Kamionkowski, Cosmological-Parameter Determination with Cosmic Microwave Background Temperature Anisotropies and Polarization.

To be published in the Proceedings of the 33rd rencontres de Moriond: Fundamental Parameters in Cosmology, Les Arcs, France, January 15-24, 1998. astro-ph/9803168.

[18] We note the similarity to the formula for the age of the universe:

$$
t_{0}=\frac{1}{H_{0}} \int_{1}^{\infty} \frac{d w}{w \sqrt{\Omega_{\Lambda}+\Omega_{C} w^{2}+\Omega_{0} w^{3}}}
$$

which is also an elliptic integral that cannot be done analytically.

[19] For a review of the cosmological constant problem see, for example,

S. Weinberg, Rev. Mod. Phys. 61, 1 (1989); Y.J. Ng, Int. J. Mod. Phys. D1, 145 (1992).

[20] S.J. Perlmutter et al.,(The Supernova Cosmology Project)

Discovery of a Supernova Explosion at Half the Age of the Universe and Its Cosmological Implications. astro-ph/9712219.

[21] S.J. Perlmutter et al.,(The Supernova Cosmology Project) astro-ph/9608199.

\section{Figure Captions.}

Fig 1. Plot of $l_{1}$ vs. $\Omega_{0}$ for $\Omega_{\Lambda}=0$.

Fig. 2. $l_{1}$ vs. $\Omega_{0}$ for the case $\Omega_{\Lambda}=1-\Omega_{0}$.

Fig. 3. Iso-l lines on $\Omega_{0}-\Omega_{\Lambda}$ plot, for (from right to left)

$l_{1}=150$ through 270 in increments $\Delta l=10$. Horizontal $=\Omega_{0}$, Vertical $=\Omega_{\Lambda}$.

Fig. 4. Three-dimensional plot of $l_{1}$ against $\Omega_{0}$ and $\Omega_{\Lambda}$. Front $=\Omega_{\Lambda}$, Right $=\Omega_{0}$. 


\section{FIGURES}

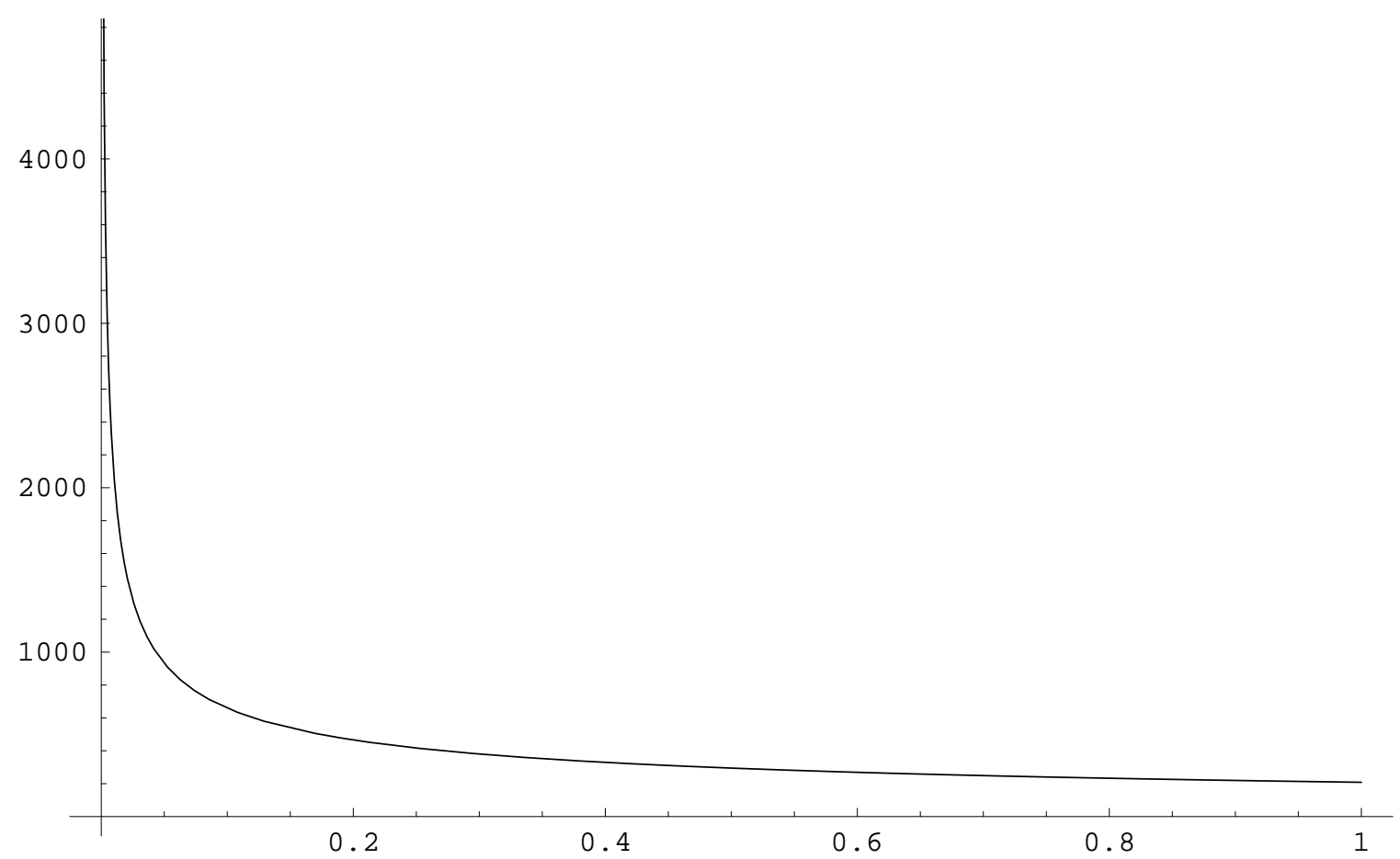

Figure 1. 


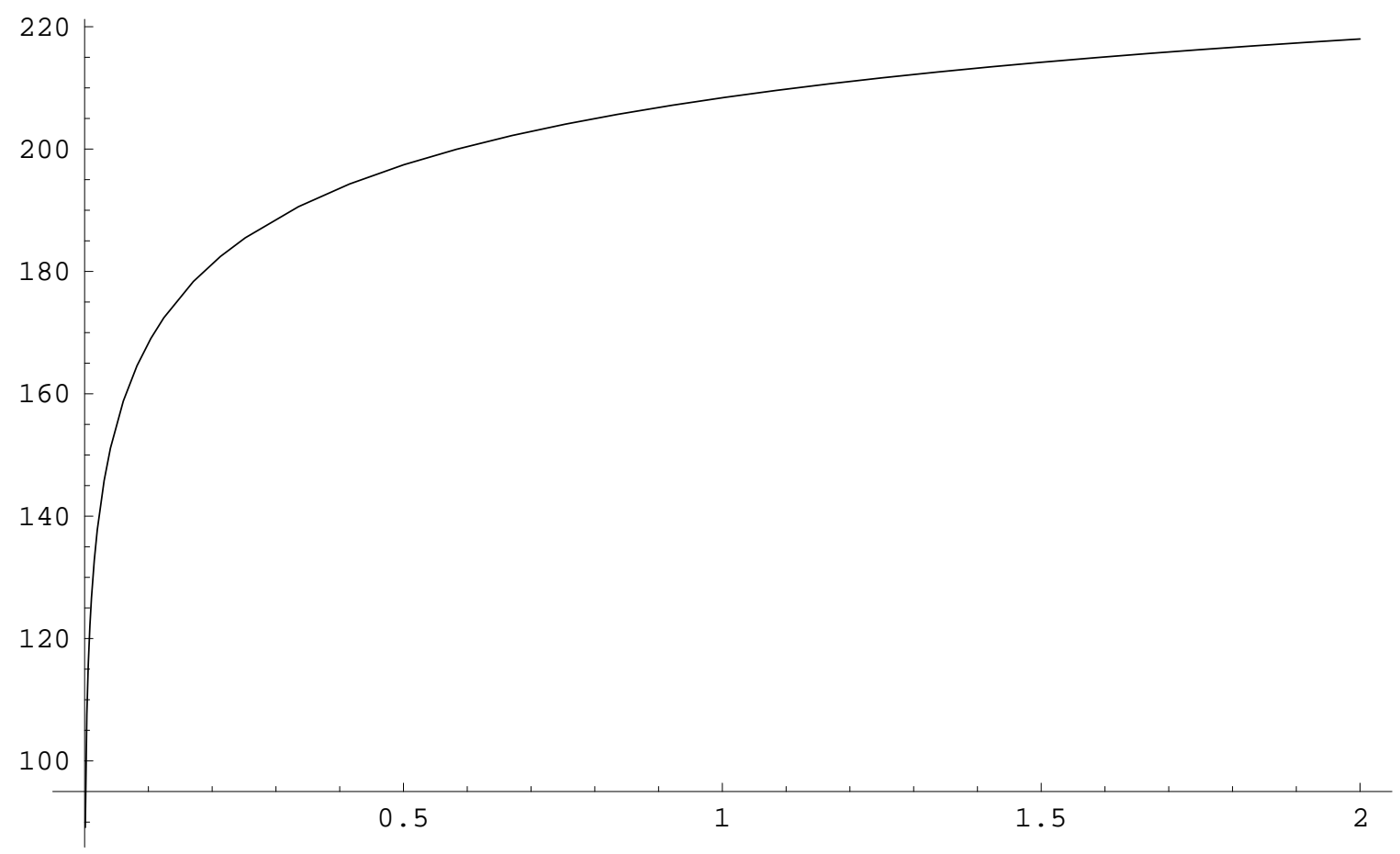

Figure 2. 


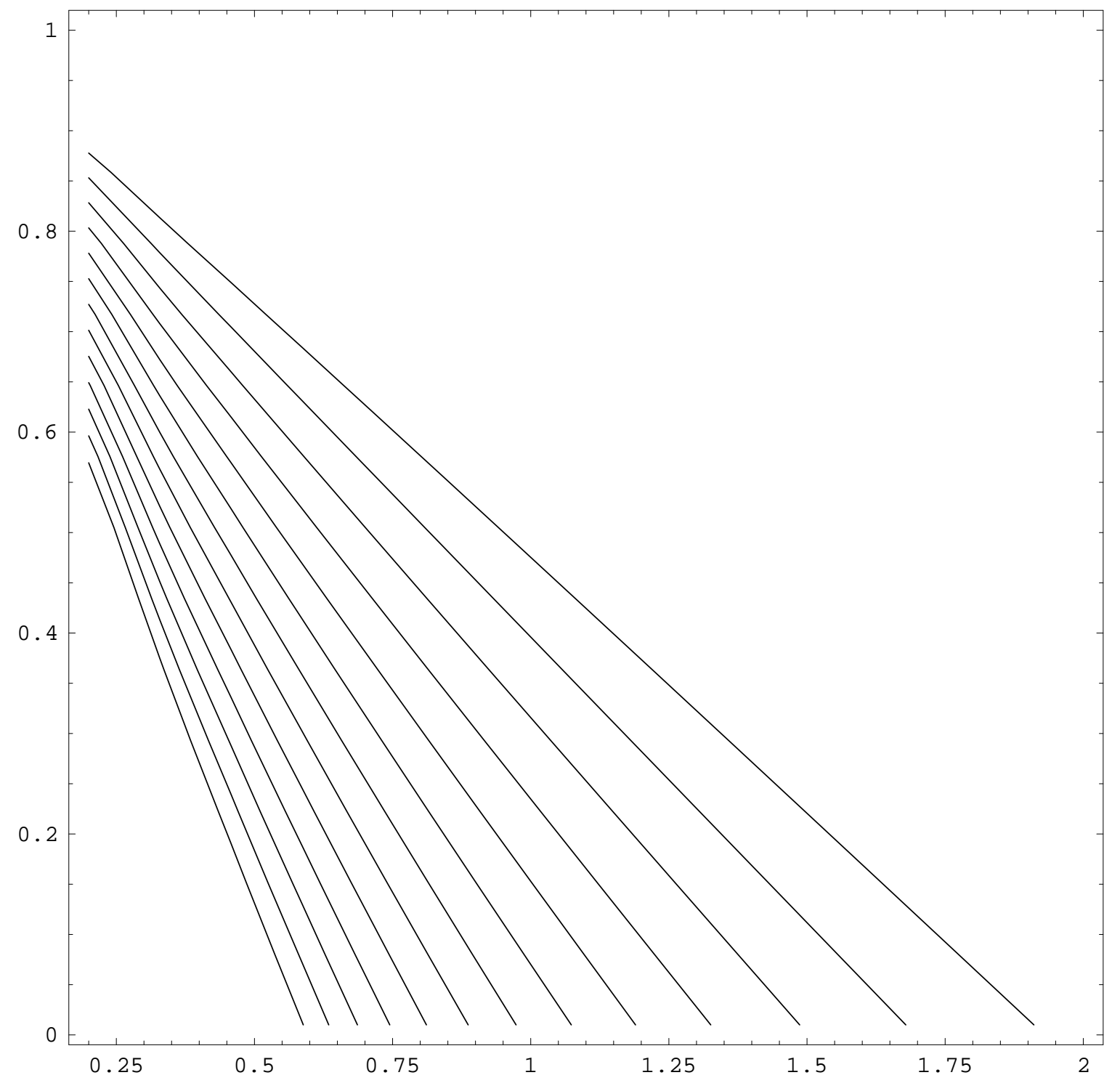

Figure 3. 


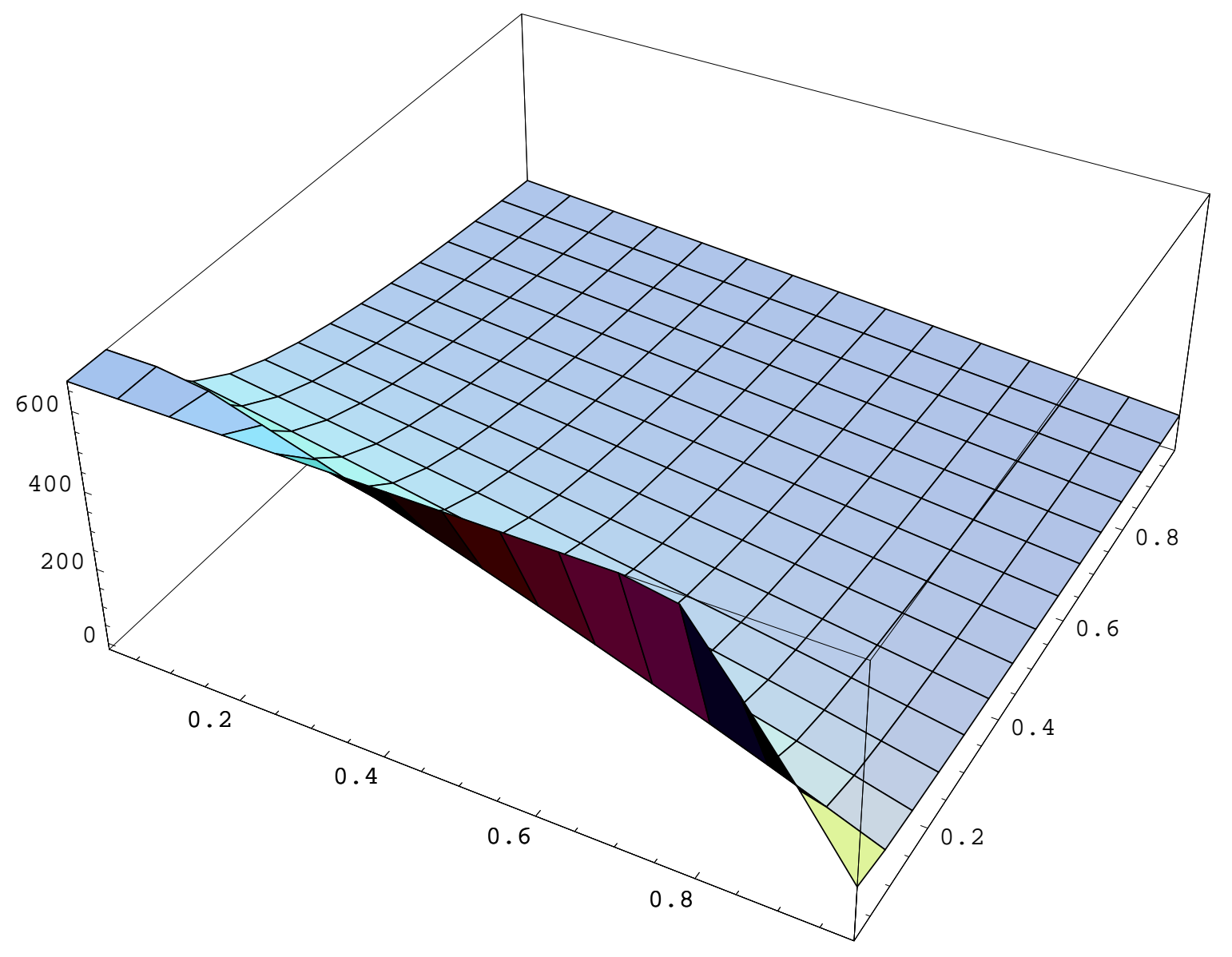

Figure 4. 